\title{
Snake venom NAD glycohydrolases: Primary structures, genomic location, and gene structure
}

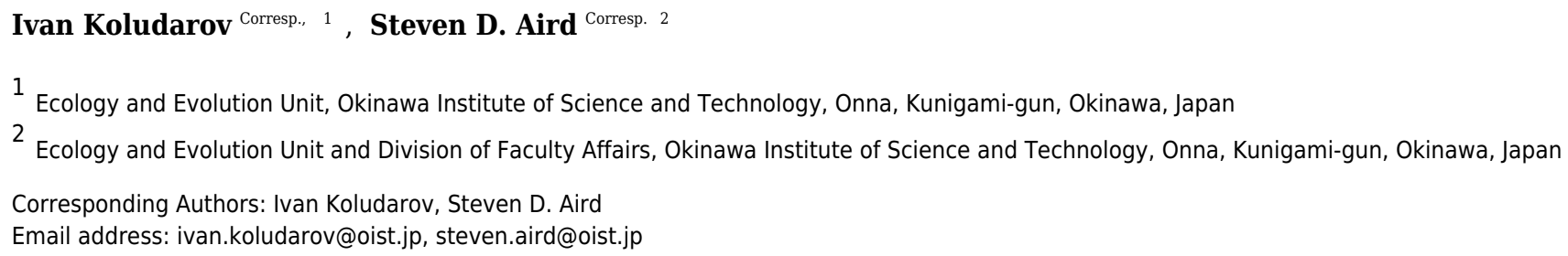

NAD glycohydrolase (EC 3.2.2.5) (NADase) sequences have been identified in 10 elapid and crotalid venom gland transcriptomes, 8 of which are complete. These sequences show very high homology, but elapid and crotalid sequences also display consistent differences. As in Aplysia kurodaiADP-ribosyl cyclase and vertebrate CD38 genes, snake venom NADase genes comprise eight exons; however, in the Protobothrops mucrosquamatus genome, the sixth exon is sometimes not transcribed, yielding a shortened NADase mRNA that encodes all six disulfide bonds, but an active site that lacks the catalytic glutamate residue. The function of this shortened protein, if expressed, is unknown. While many vertebrate $\mathrm{CD} 38 \mathrm{~s}$ are multifunctional, liberating both ADP-ribose and small quantities of cyclic ADP-ribose (CADPR), snake venom CD38 homologs are dedicated NADases. They possess the invariant TLEDTL sequence (residues 144-149) that bounds the active site and the catalytic residue, Glu228. In addition, they possess a disulfide bond (Cys121-Cys202) that specifically prevents ADP-ribosyl cyclase activity in combination with Ile224, in lieu of phenylalanine, which is requisite for ADPR cyclases. In concert with venom phosphodiesterase and 5'-nucleotidase and their ecto-enzyme homologs in prey tissues, snake venom NADases comprise part of an envenomation strategy to liberate purine nucleosides, and particularly adenosine, in the prey, promoting prey immobilization via hypotension and paralysis. 
1 Snake venom NAD glycohydrolases:

2

3

4

\title{
Primary structures, genomic location, and gene structure
}

\author{
Ivan Koludarov ${ }^{1}$ and Steven D. Aird ${ }^{2}$ \\ ${ }^{1}$ Ecology and Evolution Unit, Okinawa Institute of Science and Technology, 1919-1 Tancha, \\ Onna-son, Kunigami-gun, Okinawa, Japan 904-0495 \\ ${ }^{2}$ Division of Faculty Affairs, Okinawa Institute of Science and Technology, 1919-1 Tancha, \\ Onna-son, Kunigami-gun, Okinawa, Japan 904-0495
}

Corresponding Authors:

Steven D. Aird and Ivan Koludarov

${ }^{1}$ Ecology and Evolution Unit and ${ }^{2}$ Division of Faculty Affairs, Okinawa Institute of Science and Technology, 1919-1 Tancha, Onna-son, Kunigami-gun, Okinawa, Japan 9040495

Email addresses: steven.aird@oist.jp ivan.koludarov@oist.jp

\section{ABSTRACT}

NAD glycohydrolase (EC 3.2.2.5) (NADase) sequences have been identified in 10 elapid and crotalid venom gland transcriptomes, 8 of which are complete. These sequences show very high homology, but elapid and crotalid sequences also display consistent differences. As in Aplysia kurodai ADP-ribosyl cyclase and vertebrate CD38 genes, snake venom NADase genes comprise eight exons; however, in the Protobothrops mucrosquamatus genome, the sixth exon is sometimes not transcribed, yielding a shortened NADase mRNA that encodes all six disulfide bonds, but an active site that lacks the catalytic glutamate residue. The function of this shortened protein, if expressed, is unknown. While many vertebrate CD38s are multifunctional, liberating both ADPribose and small quantities of cyclic ADP-ribose (cADPR), snake venom CD38 homologs 
34

35

36

37

38

39

40

41

42

43

44

45

46

47

48

49

50

51

52

53

54

55

56

57

58

59

60

61

62

63

64

65

66

67

68

69

are dedicated NADases. They possess the invariant TLEDTL sequence (residues 144-149) that bounds the active site and the catalytic residue, Glu228. In addition, they possess a disulfide bond (Cys121-Cys202) that specifically prevents ADP-ribosyl cyclase activity in combination with Ile224, in lieu of phenylalanine, which is requisite for ADPR cyclases. In concert with venom phosphodiesterase and 5'-nucleotidase and their ecto-enzyme homologs in prey tissues, snake venom NADases comprise part of an envenomation strategy to liberate purine nucleosides, and particularly adenosine, in the prey, promoting prey immobilization via hypotension and paralysis.

\section{INTRODUCTION}

More than 60 years ago, Bhattacharya (1953) reported that when Bungarus fasciatus venom is incubated with NAD, it releases nicotinamide. This constituted the first evidence that some snake venoms contain an NAD glycohydrolase (NADase) (EC 3.2.2.5). However, like many other non-toxic enzymes, its presence in venoms seemed enigmatic until Aird (2002) proposed that purine nucleosides comprise core elements of the envenomation strategies of most advanced venomous snakes. Adenosine is particularly important because of its hypotensive and neuroprotective (neurosuppressive) activities. Venom NADase augments adenosine release in prey tissues by cleaving $\beta$-NAD and NADP to nicotinamide and ADP-ribose, from which adenosine can be liberated by venom and tissue phosphodiesterases in combination with venom and tissue 5'-nucleotidases. Recently, it has been reported that the Deinagkistrodon acutus NADase is capable of hydrolyzing both ATP and ADP to AMP, a function normally supplied by phosphodiesterase (Zhang et al., 2009).

Historical overview of studies on snake venom NADase

Seven years after the Bhattacharya study, Suzuki et al. (1960) examined 9 Asian snake venoms for NADase activity, using the UV detection method (340 nm) of Zatman et al. (1953) and discovered this enzyme in the venoms of Bungarus multicinctus and Trimeresurus gramineus. Venoms reported as negative for NADase included Gloydius blomhoffii, Deinagkistrodon acutus, Ovophis okinavensis, Protobothrops mucrosquamatus, Naja atra, Naja naja, and Ophiophagus hannah.

In a study of 37 elapid, viperid and crotalid venoms, also using UV detection, Tatsuki et al. (1975) confirmed the earlier findings of NADase activity in venoms of the two Bungarus species and further identified it in venoms of Agkistrodon c. contortrix, A. c. mokasen, A. c. laticinctus, A. p. piscivorus, Gloydius blomhoffii, Deinagkistrodon acutus, and Causus rhombeatus, the first viperid examined. All other taxa were reportedly negative for 
70 NADase activity (Table 1). Using a succession of five liquid chromatographic procedures

71 they isolated the enzyme from G. blomhoffii venom and characterized it biochemically. 72 The Gloydius enzyme readily hydrolyzed $\beta$-NAD and NADP+, and cleaved 373 acetylpyridine adenine dinucleotide, but it did not hydrolyze NADH, NADPH, $\alpha$-NAD+, 74 or $\beta$-nicotinamide mononucleotide ( $\beta$-NMN) (Tatsuki et al., 1975). They did not estimate 75 the enzyme's molecular weight.

Yost and Anderson (1981) characterized the NADase from Bungarus fasciatus venom, and reported that it was a homodimeric glycoprotein of 120-130,000 Da, having a monomeric molecular weight of 62,000 (denaturing SDS PAGE). The enzyme comprised approximately $0.1 \%$ of Bungarus fasciatus venom by mass (Yost \& Anderson, 1981; Anderson, Yost \& Anderson, 1986) while a value of $0.5 \%$ was reported from Deinagkistrodon acutus venom (Wu et al., 2002) using a simpler chromatographic procedure with more sophisticated resins.

Huang et al. (1988) investigated the NADase from Deinagkistrodon acutus venom. That enzyme is a homodimeric glycoprotein of about 98,000 Da, having a minimum monomeric molecular mass of $33,500 \mathrm{Da}$, allowing for a carbohydrate content estimated at $33 \%$. The authors reported that the N-terminal amino acid was proline. As with the Gloydius enzyme (Tatsuki et al., 1975), NADP was the optimal substrate (Huang et al., 1988). The Deinagkistrodon NADase is a metalloenzyme, containing a single, essential copper ion.

Despite these studies, no structural information has been reported for any snake venom NADase. Because our group has completed a series of elapid and crotalid venom gland transcriptomic studies employing high-throughput techniques (Aird et al., 2013, 2015, $2017 \mathrm{~b}$ ), we searched these transcriptomes for the presence of NAD glycohydrolase. It was found in all of them, and we here report their primary structures, possible 3D structures, genomic arrangement, and gene structure.

\section{MATERIALS \& METHODS}

The NADase sequences examined were generated in previously reported studies (Aird et al., 2013, 2015, 2017b). Models of the Micrurus surinamensis and Protobothrops 
104 2012) (http://galaxy.seoklab.org/cgi-bin/submit.cgi?type=TBM). Three-dimensional 105 structures were visualized and analyzed using Chimera version 1.13 (Pettersen et al., 106 2004) (http://www.rbvi.ucsf.edu/chimera). Probable disulfide bonds were created 107 manually based upon cysteine locations and energy minimizations were performed 108 thereafter to optimize the structures. Hydrophilicity/hydrophobicity scores were calculated using the Gravy calculator at: http://www.gravy-calculator.de

110

111 Geneious 8.1.9 (https://www.geneious.com) was used to BLAST elapid and crotalid 112 transcriptomes for NAD glycohydrolase sequences using Gallus gallus CD38 as a query 113 sequence and to align snake venom sequences (Table 2). Genomic scaffolds were 114 examined with ncbi-blast/2.7.1+ suite (https://blast.ncbi.nlm.nih.gov) using Homo sapiens 115 CD38 and Protobothrops mucrosquamatus CD38 as queries and further analyzed in 116 Geneious 8.1.9 to determine exon sequences (Table 2).

\section{RESULTS \& DISCUSSION}

\section{Snake Venom NADase Amino Acid Sequences}

121

The NCBI Protein site was searched for vertebrate NAD glycohydrolase sequences and the sequence of chicken ADP-ribosyl cyclase (ADQ89191.1), also known as CD38, was downloaded for use as a query sequence. TBLASTN searches of venom gland transcriptomes of 10 elapid and crotalid species were performed using Geneious 8.1.9. A highly similar sequence was identified in each transcriptome, 8 of which were complete (Figure 1), and NADase transcripts were present at low levels in venom gland transcriptomes of all 30 Protobothrops mucrosquamatus examined by (Aird et al., 2017a). A partial (30-residue), unidentified sequence also occurs in the Ophiophagus hannah genome (L345_15802). The former sequences were aligned with CD38 sequences from Gallus gallus, Xenopus laevis, Anolis carolinensis, and Homo sapiens, using Geneious (Figure 1).

None of the venom NADases appears to have a signal peptide, based upon sequence analyses using SignalP 4.1 (Petersen et al., 2011). All are readily distinguished from the former four vertebrate $\mathrm{CD} 38$ sequences, as all venom sequences commence with the $\mathrm{N}$ terminal sequence, MPFQNS, rather than with proline, as reported by Huang et al. (1988). Like other vertebrate CD38 sequences, snake venom sequences possess a hydrophilic 14residue $\mathrm{N}$-terminus (Figure 1). In all NADases, immediately C-terminal to this hydrophilic block, there is a hydrophobic, 25-amino acid segment containing 17 aliphatic residues ( $\mathrm{L}, \mathrm{V}, \mathrm{I}$, and $\mathrm{G}), 3$ threonine residues, 1 lysine, and 2-3 phenylalanines. These are 
140 followed by another 20 residues that are nearly all hydrophilic. The aliphatic segment

141 almost has the appearance of a signal peptide (Figure 1).

142

143 Venom NADases are also clearly resolvable into elapid and crotalid sequences. The

144 Micrurus NADases have 300 amino acids, while crotalid enzymes have 302-303 and may

145 be readily distinguished based upon sequence differences at various positions.

146 Coralsnake and pitviper sequences display the following respective differences: Q/E52,

147 R or Q/W91, S/R105, H/N145, N/D146, K/N167, D/N170, E/M218, I/T217, N/S219, Q/K224,

148 K/E240, and probably also G/D253, S/N276, I/S278, T/A301, and T/K303, although the

149 Ovophis okinavensis and Protobothrops flavoviridis sequences are incomplete at this point

150 (numbered as in Figure 1). At several positions, some Micrurus sequences show the

151 crotalid residue while others have a different amino acid.

152

The M. surinamensis apoprotein has a monomeric molecular weight of 34,206 Da and a predicted $\mathrm{pI}$ of 7.93, using the Expasy Compute $\mathrm{pI} / \mathrm{MW}$ tool. Accordingly, the coralsnake enzymes have masses only slightly higher than the 33,500 predicted for the Deinagkistrodon enzyme (Huang et al., 1988). The Micrurus sequences contain three potential N-glycosylation sites (NKSL, position 125; NGSI, position 214; NRSI, position 274), based upon results obtained using the Expasy NetNGlyc 1.0 Server (http://www.cbs.dtu.dk/services/NetNGlyc/). The first, which is common to elapid and crotalid sequences alike (Figure 1), has the highest likelihood of being glycosylated, although it may not be since venom NADases lack signal peptides. Nonetheless, Huang et al. (1988) reported a carbohydrate content of about 33\% for the Deinagkistrodon NADase, so it seems likely that at least the NKSL at position 125 is N-glycosylated. Based upon analysis with the Expasy NetOGlyc server, there are no probable O-glycosylation sites (Steentoft et al., 2013).

Higher-level structural attributes of vertebrate NADases

167 Human CD38 is an ecto-enzyme with a long, helical membrane anchor and an intracellular N-terminal segment believed to be a random coil (Malavasi et al., 1992; Prasad et al., 1996; Lee, 2006) (Figure 2). In addition to its enzymatic activity, CD38 also transduces signals to the cytoplasm. It is thought to regulate metabolism and participates in the pathogenesis of diverse maladies such as inflammation, obesity, diabetes, heart disease, asthma, and aging (Chini et al., 2018). Its enzymatic activity is involved in many of these functions. Moreover, CD38 has been identified as a cell-surface marker in multiple myeloma and other blood-related cancers (Chini et al., 2018).

176 In contrast, snake venom enzymes are soluble rather than membrane-bound (Tatsuki et 177 al., 1975; Yost \& Anderson, 1981), like the Aplysia ADP-ribosyl cyclase (Lee \& Aarhus, 
178 1991). While venom NADases could conceivably be membrane-bound in exosomes, they

179 have not been reported as exosomal enzymes (Ogawa et al., 2008), and their elution on

180 Sephadex G-100 is appropriate for soluble enzymes of $\sim 100 \mathrm{kDa}$ rather than for exosomes

181 (Tatsuki et al., 1975). Exosomal embedding seems further unlikely in that all of the venom

182 NADases reported here possess a very short N-terminal $\alpha$-helix and a random coil,

183 instead of the long $\alpha$-helical membrane anchor of human CD38 (Lee, 2006) (Figure 2A).

184 Snake venom NADase residues 45-302 superimpose almost perfectly upon the crystal

185 structure of the soluble extracellular domain of human CD38, except for the divergent C-

186 termini and the truncated N-termini (3F6Y_A) (Figure 2B).

187

188 Yost and Anderson (1981) found that the Bungarus NADase is a homodimer with a 189 dimeric mass of $125-130 \mathrm{kDa}$, and a subunit mass of $62 \mathrm{kDa}$ on reducing SDS-PAGE. 190 Huang et al.(1988) reported slightly lower values of $98 \mathrm{kDa}$ and $50 \mathrm{kDa}$ for the

191 Deinagkistrodon enzyme, but apparently employed non-reducing SDS-PAGE, suggesting

192 that the homodimer in that taxon is non-covalent. Interestingly, our Micrurus sequences

193 have 12 cysteine residues, arranged in the 3D structure in a manner consistent with 6

194 disulfide bonds (C68-C84; C101-C181; C121-C202; C162-C175; C256-C277; C289-C298)

195 (Figure 2) (Egea et al., 2012), coinciding exactly with the disulfide bonds in CD38 (Figure

196 2C); however, the two Protobothrops sequences both have a 13th cysteine in the

197 penultimate position (C-terminus), such that that the Protobothrops enzymes could be

198 covalent homodimers (Figure 1).

199

200 The N-terminal 50 residues of the M. surinamensis NADase are slightly hydrophobic, 201 with a Gravy score of 0.142 , while the N-terminal 50 residues of human CD38 202 [BAA18966.1] have a significantly more hydrophobic Gravy score of 0.710. As a result, 203 the soluble venom enzymes appear to have a slightly more compact $\mathrm{N}$-terminal domain 204 than human CD38 (Figure 2D).

205

206 Many invertebrate and vertebrate enzymes exhibiting NADase activity are 207 multifunctional, not only hydrolyzing $\beta-\mathrm{NAD}(\mathrm{P})^{+}$to nicotinamide and ADP-ribose, but 208 also exhibiting ADP- and GDP-ribosyl cyclase, and cADPR/cGDPR hydrolase activities 209 (Howard et al., 1993; Lee, Graeff \& Walseth, 1997; Ziegler et al., 1997; Ziegler, Jorcke \& 210 Schweiger, 1997; Augustin, Muller-Steffner \& Schuber, 2000; Ferrero et al., 2014).

211 However, unlike ADP-ribosyl cyclase, human CD38 converts very little $\beta$-NAD to

212 cADPR (Lee, 2006).

213

214 In contrast to many invertebrate and vertebrate CD38 homologs, Yost and Anderson 215 (1981) found that when $\beta$-NAD was hydrolyzed by Bungarus fasciatus NADase, 216 nicotinamide and ADP-ribose were the sole products. The B. fasciatus enzyme does not 217 catalyze the conversion of $\beta$-NAD to cyclic ADP-ribose (cADPR). This lack of cyclase 
218 activity results in part from the presence of a disulfide bond (C124-C206 in Figure 1;

219 C121-C202, actual) which is absent in Aplysia ADP-ribosyl cyclase (Tohgo et al., 1994).

220 Moreover, this disulfide bond is present in all snake venom NADases for which we have

221 sequences. Graeff et al. (2009) reported that mutation of Phe221 in Aplysia ADP-ribosyl

222 cyclase (Phe227 in Figure 1) reduced cADPR production and increased ADPR liberation.

223 Consistent with this conclusion, all snake NADases and at least some vertebrate CD38's

224 have isoleucine in this position (Figure 1), effectively preventing cADPR formation.

225 Snake venom NADases also all have the conserved TLEDTL (144-149) sequence (Figure

226 2A) (residues 149-154 in Figure 1) that forms the bottom of the active site pocket (Lee,

227 2006), and the catalytic residue, Glu226 (Glu232 in Figure 1), which are present in all 228 CD38 molecules (Graeff et al., 2001). Substitution of Glu-146 with Phe, Asn, Gly, Asp,

229 Leu, or Ala resulted in cyclase activity up to 9x higher than of wild-type CD38 (Graeff et

230 al., 2001).

231

232 Genome location of vertebrate NAD glycohydrolases/ADP-ribosyl cyclases

233 We performed genome-wide BLAST searches to locate the NAD glycohydrolase gene in 234 the genomes of Homo sapiens, Gallus gallus, Alligator mississippiensis, Anolis carolinensis,

235 Protobothrops mucrosquamatus, Python bivitattus, Thamnophis sirtalis, and Xenopus laevis.

236 After locating the genes, we manually checked their sequences and compared them with

237

238

239 In the genomes surveyed, NAD glycohydrolase, $C D 38$, is located in the vicinity of the 240 CC2D2A and PROM1 genes (Ch1L in Xenopus laevis, Ch4 in Homo sapiens and Gallus 241 gallus), usually directly downstream from the FGFBP1 gene. Non-squamate vertebrates 242 have a duplicate gene, called BST1, located upstream from CD38. Squamates apparently 243 lack BST1 in this region, probably due to clade-specific gene loss.

244

245

246

247

248

249

250

251

252

253

254

\section{Gene structure of vertebrate NAD glycohydrolases/ADP-ribosyl cyclases}

Human CD38 displays similar intron-exon architecture to that seen in the invertebrate, Aplysia kurodai ADP-ribosyl cyclase (Nata et al., 1995), suggesting that this architecture is highly conserved. It comprises 8 exons, extending more than $77 \mathrm{~kb}$ in the human genome (Nata et al., 1997). However, in Protobothrops mucrosquamatus (and presumably in other venomous snakes) the 8-exon gene is transcribed in two variants, a long NADase similar to CD38 of other vertebrates (Figure 3), and a shorter form that lacks exon 6 (Figure 4). Exon 6 contains no cysteines, and its deletion results in the loss of 31 amino acids from Ser223-Gln253 (Figure 4A). Owing to the presence of split arginine codons at both the exon 6-7 and exon 7-8 boundaries, no amino acid substitution occurs in the shortened (272-residue) structure. Instead, the effect of removing these 31 residues is to delete the 
255 loose helical region (Ser223-Gln253) from the center of the molecule, including the 256 essential catalytic residue, Glu229 (Figure 4A; Glu232 in Figure 1), while leaving the

257

258

259

260

261

262

263

264

265

266

267

268

269

270

271

272

273

274

275

276

277

278

279

280

281

282

283

284

285

286

287

288

289

290

291 remainder of the structure essentially intact (Figure 4B). If this protein is expressed, it is difficult to imagine what its function might be, but with its precise alternate splicing, this does not seem like a pseudogene.

\section{CONCLUSIONS}

All snake venom gland transcriptomes and venomous snake genomes published to date contain sequences for NAD glycohydrolases, pointing to a significant role in envenomation. At least some crotalids may produce two forms of this enzyme. Strategically the function of snake venom NADases is to drive the release of adenosine from NADP and $\beta$-NAD in prey tissues and to block its conversion to cADPR. Guanosine is also released from NGD. Both purines contribute to prey immobilization via hypotension/circulatory shock and paralysis caused by neurosuppression (Aird, 2002, 2009).

\section{ACKNOWLEDGEMENTS}

We thank Alexander S. Mikheyev for critical reading of the manuscript that substantially improved its organization.

\section{REFERENCES}

Aird SD. 2002. Ophidian envenomation strategies and the role of purines. Toxicon: official journal of the International Society on Toxinology 40:335-393.

Aird SD. 2009. The Role of Purine and Pyrimidine Nucleosides in Snake Venoms. In: Mackessy SP ed. Handbook of Venoms and Toxins of Reptiles. Boca Raton, FL: CRC Press, 393-419.

Aird SD., Aggarwal S., Villar-Briones A., Tin MM-Y., Terada K., Mikheyev AS. 2015. Snake venoms are integrated systems, but abundant venom proteins evolve more rapidly. BMC genomics 16:647.

Aird SD., Arora J., Barua A., Qiu L., Terada K., Mikheyev AS. 2017a. Population Genomic Analysis of a Pitviper Reveals Microevolutionary Forces Underlying Venom Chemistry. Genome biology and evolution 9:2640-2649.

Aird SD., Silva NJ da Jr., Qiu L., Villar-Briones A., Saddi VA., Pires de Campos Telles M., Grau ML., Mikheyev AS. 2017b. Coralsnake Venomics: Analyses of Venom Gland Transcriptomes and Proteomes of Six Brazilian Taxa. Toxins 9:1-64. 
292

293

294

295

296

297

298

299

300

301

302

303

304

305

306

307

308

309

310

311

312

313

314

315

316

317

318

319

320

321

322

323

324

325

326

327

328

329

330

331

Aird SD., Watanabe Y., Villar-Briones A., Roy MC., Terada K., Mikheyev AS. 2013. Quantitative high-throughput profiling of snake venom gland transcriptomes and proteomes (Ovophis okinavensis and Protobothrops flavoviridis). BMC genomics 14:790.

Anderson BM., Yost DA., Anderson CD. 1986. Snake venom NAD glycohydrolase: purification, immobilization, and transglycosidation. Methods in enzymology 122:173181.

Augustin A., Muller-Steffner H., Schuber F. 2000. Molecular cloning and functional expression of bovine spleen ecto-NAD+ glycohydrolase: structural identity with human CD38. Biochemical Journal 345 Pt 1:43-52.

Bhattacharya KL. 1953. Effect of snake venoms on coenzyme-I. Journal of the Indian Chemical Society 30:685.

Chini EN., Chini CCS., Espindola Netto JM., de Oliveira GC., van Schooten W. 2018. The Pharmacology of CD38/NADase: An Emerging Target in Cancer and Diseases of Aging. Trends in pharmacological sciences 39:424-436.

Egea PF., Muller-Steffner H., Kuhn I., Cakir-Kiefer C., Oppenheimer NJ., Stroud RM., Kellenberger E., Schuber F. 2012. Insights into the mechanism of bovine CD38/NAD+glycohydrolase from the X-ray structures of its Michaelis complex and covalently-trapped intermediates. PloS one 7:e34918.

Ferrero E., Lo Buono N., Horenstein AL., Funaro A., Malavasi F. 2014. The ADP-ribosyl cyclases--the current evolutionary state of the ARCs. Frontiers in bioscience 19:9861002.

Graeff R., Liu Q., Kriksunov IA., Kotaka M., Oppenheimer N., Hao Q., Lee HC. 2009. Mechanism of Cyclizing NAD to Cyclic ADP-ribose by ADP-ribosyl Cyclase and CD38. The Journal of biological chemistry 284:27629-27636.

Graeff R., Munshi C., Aarhus R., Johns M., Lee HC. 2001. A single residue at the active site of CD38 determines its NAD cyclizing and hydrolyzing activities. The Journal of biological chemistry 276:12169-12173.

Howard M., Grimaldi JC., Bazan JF., Lund FE., Santos-Argumedo L., Parkhouse RM., Walseth TF., Lee HC. 1993. Formation and hydrolysis of cyclic ADP-ribose catalyzed by lymphocyte antigen CD38. Science 262:1056-1059.

Huang WZ., Wang C., Luo LQ., Lu ZX. 1988. Properties of NAD glycohydrolase purified from five-pace snake (Agkistrodon acutus) venom. Toxicon 26:535-542.

Ko J., Park H., Heo L., Seok C. 2012. GalaxyWEB server for protein structure prediction and refinement. Nucleic acids research 40:W294-7.

Lee HC. 2006. Structure and enzymatic functions of human CD38. Molecular medicine 12:317-323.

Lee HC. 2006. Structure and enzymatic functions of human CD38. Molecular medicine 12:317-323.

Lee HC., Aarhus R. 1991. ADP-ribosyl cyclase: an enzyme that cyclizes NAD+ into a 
calcium-mobilizing metabolite. Cell regulation 2:203-209.

333

334

335

336

337

338

339

340

341

342

343

344

345

346

347

348

349

350

351

352

353

354

355

356

357

358

359

360

361

362

363

364

365

366

367

368

369

370

371

Lee HC., Graeff RM., Walseth TF. 1997. ADP-Ribosyl Cyclase and CD38. In: Advances in Experimental Medicine and Biology. 411-419.

Magdeldin S., Yoshida Y., Li H., Maeda Y., Yokoyama M., Enany S., Zhang Y., Xu B., Fujinaka H., Yaoita E., Sasaki S., Yamamoto T. 2012. Murine colon proteome and characterization of the protein pathways. BioData mining 5:11.

Malavasi F., Funaro A., Alessio M., DeMonte LB., Ausiello CM., Dianzani U., Lanza F., Magrini E., Momo M., Roggero S. 1992. CD38: a multi-lineage cell activation molecule with a split personality. International journal of clinical \& laboratory research 22:73-80.

Nata K., Sugimoto T., Tohgo A., Takamura T., Noguchi N., Matsuoka A., Numakunai T., Shikama K., Yonekura H., Takasawa S. 1995. The structure of the Aplysia kurodai gene encoding ADP-ribosyl cyclase, a second-messenger enzyme. Gene 158:213-218.

Nata K., Takamura T., Karasawa T., Kumagai T., Hashioka W., Tohgo A., Yonekura H., Takasawa S., Nakamura S., Okamoto H. 1997. Human gene encoding CD38 (ADPribosyl cyclase/cyclic ADP-ribose hydrolase): organization, nucleotide sequence and alternative splicing. Gene 186:285-292.

Ogawa Y., Kanai-Azuma M., Akimoto Y., Kawakami H. 2008. Exosome-like vesicles in Gloydius blomhoffii blomhoffii venom. Toxicon: official journal of the International Society on Toxinology.

Petersen TN., Brunak S., von Heijne G., Nielsen H. 2011. SignalP 4.0: discriminating signal peptides from transmembrane regions. Nature methods 8:785-786.

Pettersen EF., Goddard TD., Huang CC., Couch GS., Greenblatt DM., Meng EC., Ferrin TE. 2004. UCSF Chimera--a visualization system for exploratory research and analysis. Journal of computational chemistry 25:1605-1612.

Steentoft C., Vakhrushev SY., Joshi HJ., Kong Y., Vester-Christensen MB., Schjoldager KT-BG., Lavrsen K., Dabelsteen S., Pedersen NB., Marcos-Silva L., Gupta R., Bennett EP., Mandel U., Brunak S., Wandall HH., Levery SB., Clausen H. 2013. Precision mapping of the human O-GalNAc glycoproteome through SimpleCell technology. The EMBO journal 32:1478-1488.

Suzuki T., Iizuka K., Murata Y. 1960. Studies on Snake Venom. IX. On the studies of diphosphopyridine nucleotidase in snake venom. Yakugaku zasshi: Journal of the Pharmaceutical Society of Japan 80:868-875.

Tatsuki T., Iwanaga S., Oshima G., Suzuki T. 1975. Snake venom NAD nucleosidase: its occurrence in the venoms from the genus Agkistrodon and purification and properties of the enzyme from the venom of A. halys blomhoffii. Toxicon: official journal of the International Society on Toxinology 13:211-220.

Tohgo A., Takasawa S., Noguchi N., Koguma T., Nata K., Sugimoto T., Furuya Y., Yonekura H., Okamoto H. 1994. Essential cysteine residues for cyclic ADP-ribose synthesis and hydrolysis by CD38. The Journal of biological chemistry 269:28555-28557. Wu SD., Liu Y., Xu X., Zhu Z. 2002. Purification of NAD glycohydrolase from 
372 Agkistrodon acutus venom. Protein expression and purification 25:319-322.

373 Yost DA., Anderson BM. 1981. Purification and properties of the soluble NAD

374 glycohydrolase from Bungarus fasciatus venom. The Journal of biological chemistry 375 256:3647-3653.

376 Zatman LJ., Kaplan NO., Colowick SP. 1953. Inhibition of spleen diphosphopyridine 377 nucleotidase by nicotinamide, an exchange reaction. The Journal of biological chemistry 378 200:197-212.

379 Zhang L., Xu X., Luo Z., Shen D., Wu H. 2009. Identification of an unusual AT(D)Pase380 like activity in multifunctional NAD glycohydrolase from the venom of Agkistrodon 381 acutus. Biochimie 91:240-251.

382 Ziegler M., Jorcke D., Herrero-Yraola A., Schweiger M. 1997. Bovine liver mitochondrial 383 NAD+ glycohydrolase. Relationship to ADP-ribosylation and calcium fluxes. 384 Advances in experimental medicine and biology 419:443-446.

385 Ziegler M., Jorcke D., Schweiger M. 1997. Metabolism of cyclic ADP-ribose: a new role 386 for NAD+ glycohydrolases. Reviews of physiology, biochemistry and pharmacology 387 131:89-126. 


\section{Figure 1}

Alignment of 10 snake venom NADases with human, frog, and chicken CD38 sequences.

The snake NADase sequences lack signal peptides. They are readily distinguished from the former three sequences, and are furthermore clearly resolvable as elapid and crotalid sequences, based upon sequence differences at various positions. Like their mammalian homologs, Micrurus sequences have 302 amino acids, including 12 cysteines, presumably arranged in 6 disulfide bonds (Egea et al. 2012). The two complete Protobothrops sequences have 13 cysteines, like the chicken enzyme, raising the possibility of a covalent homodimer. The truncated C-terminus of the $P$. flavoviridissequence is almost certainly a misassembly or less likely, a pseudogene, since the last 14 residues (226-239) and the premature stop codon bear no resemblance to the other sequences. Asterisks denote stop codons. Accession numbers for other vertebrate sequences: Homo sapiens, BAA18966.1; Xenopus laevis, BAL72804.1; Gallus gallus, ADQ89191.1; Anolis carolinensis, XP_016848812.1. Amino acid color scheme: Orange, methionine; Yellow, cysteine; Pink, proline; Gray, aliphatic; Purple, aromatic; Royal Blue, basic; Red, acidic; Green, hydroxylated; Sky Blue, amidated. 

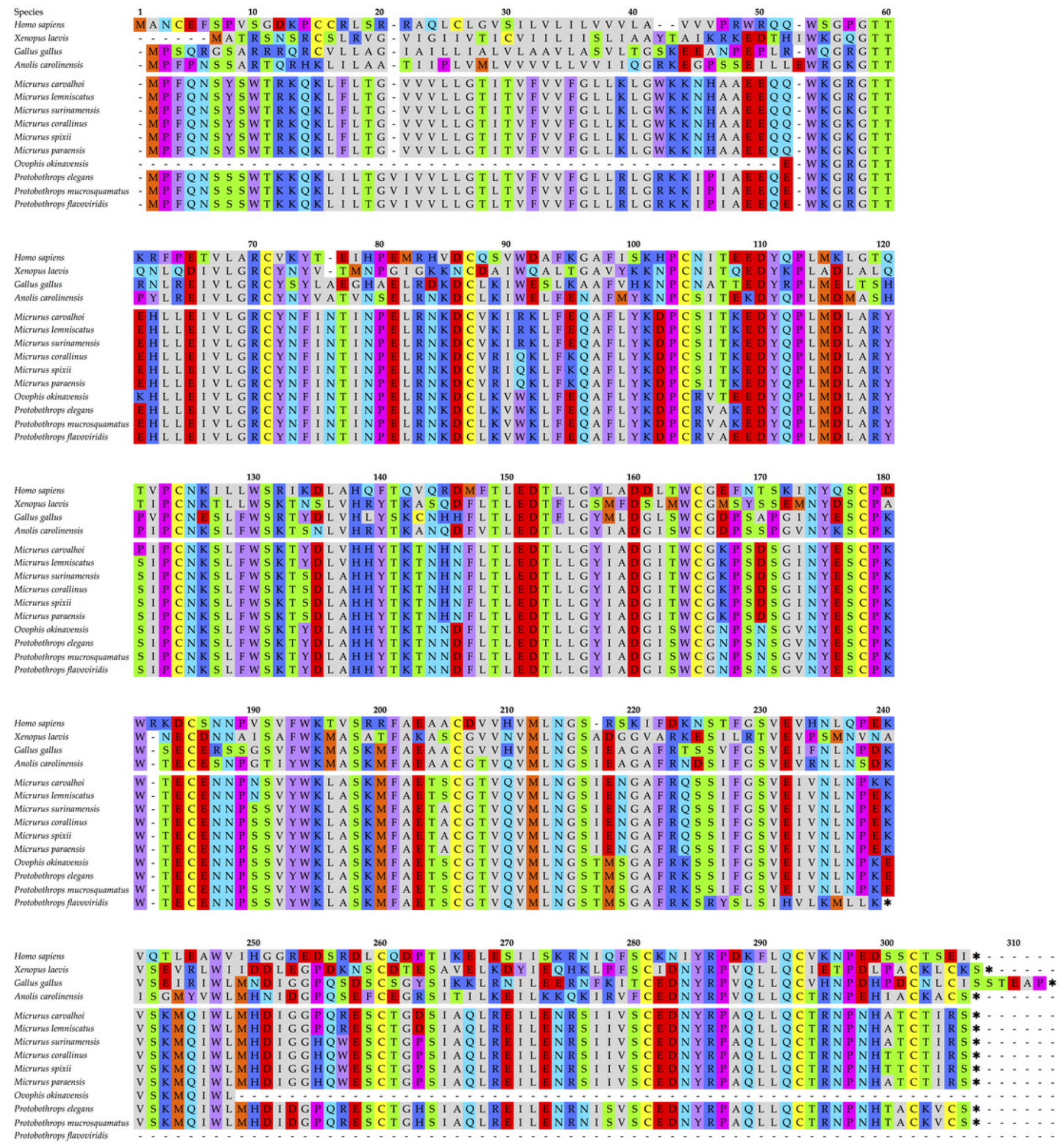


\section{Figure 2 (on next page)}

The predicted 3D structure of a soluble venom NADases is more compact than that of its membrane-bound human homolog, owing to its somewhat condensed, soluble $\mathrm{N}$ terminus

The enzyme from Micrurus surinamensis venom (left) is compared with human CD38 (right).

A. Venom enzymes lack the membrane-spanning alpha-helix and the intracellular domain (residues 1-44) of human CD38. Venom enzymes possess a soluble random coil instead. The two models are shown as ribbon structures with rainbow coloring to distinguish different regions from the $\mathrm{N}$ - (blue) to the $\mathrm{C}$-terminus (red). B. Superimposed portions of the two structures, corresponding to the soluble domain of human $C D 38$, modeled upon the crystal structure of the soluble domain of human CD38 (3F6Y_A). The two structures superimpose almost perfectly except for the divergent C-termini and the truncated N-termini. Residues 45300 of the M. surinamensis NADase (red) and residues 45-296 of human CD38 (blue) are shown. Left, frontal view; Right, left-side view. C. Disulfide bonds are identical in theM. surinamensis NADase and human CD38. The Cys121-Cys202 disulfide bond, in combination with other residues, helps to prevent ADP-ribosyl cyclase activity, forcing the conversion of $\beta$ NAD and other suitable substrates to ADP-ribose, which is subsequently hydrolyzed to adenosine, the strategic target. D. The M. surinamensis NADase and human CD38 are both hydrophilic overall, with Gravy scores of -0.292 and -0.306 , respectively. However, while the $\mathrm{N}$-terminal 44 residues of the $M$. surinamensis NADase are slightly hydrophobic (0.142), the $\mathrm{N}$-terminus of CD38 is strongly hydrophobic (0.710). Gravy scores below 0 are more likely globular, hydrophilic proteins, while scores above 0 are more likely membrane-bound and hydrophobic (Magdeldin et al., 2012). Surfaces are rendered to show Kyte-Doolittle hydrophobicity with blue residues being most hydrophilic and red residues being most hydrophobic. Models were created with GalaxyTBM using human CD38 (3F6Y) as a template (Ko et al. 2012) . Disulfide bond formation, energy minimization, and structural manipulations were performed using Chimera 1.13 (Pettersen et al. 2004). 


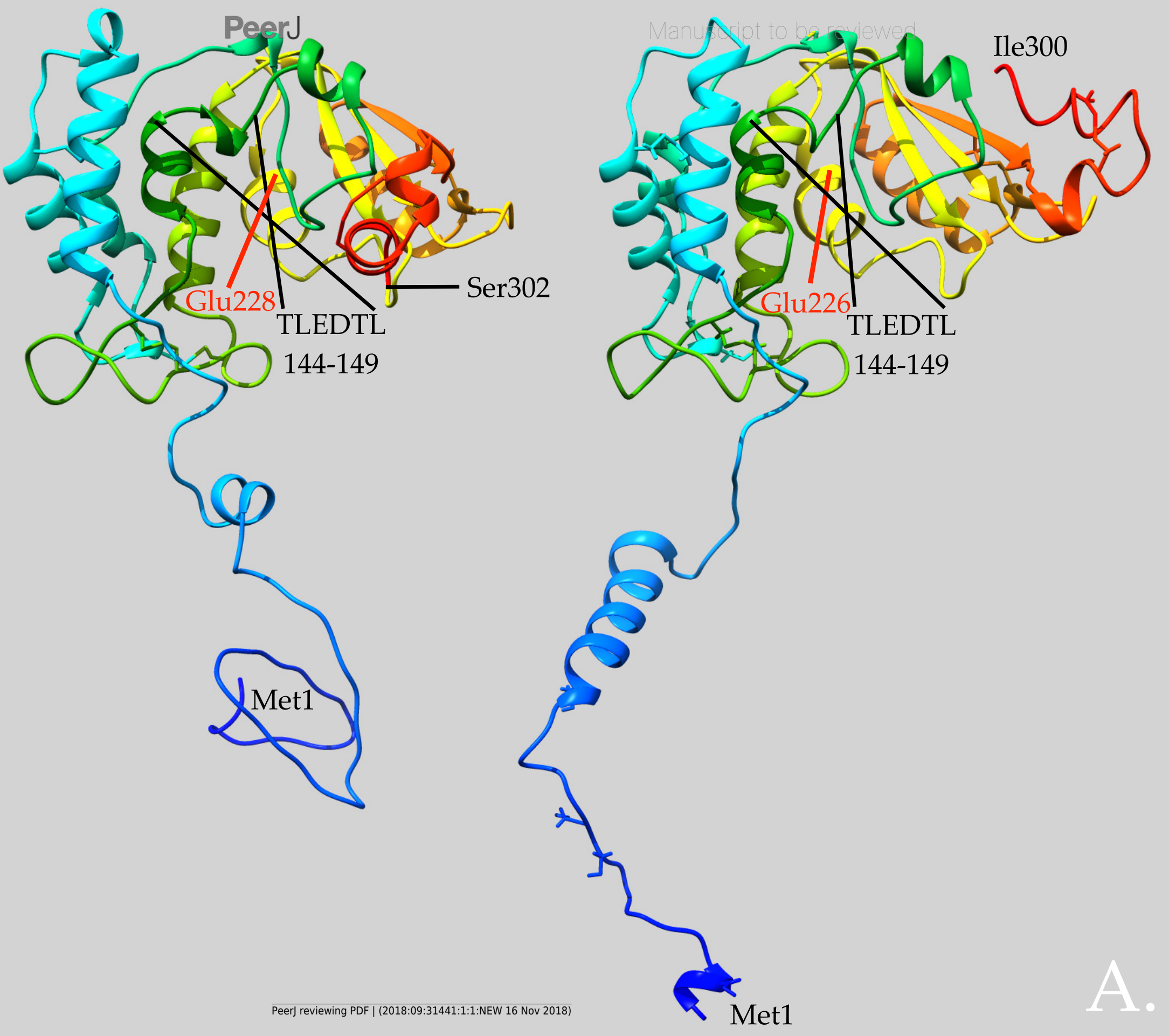




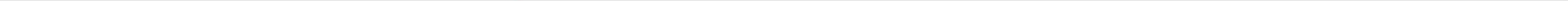




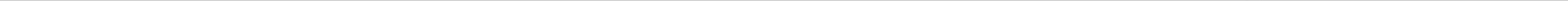


PeerJ

Manuscript to be reviewed
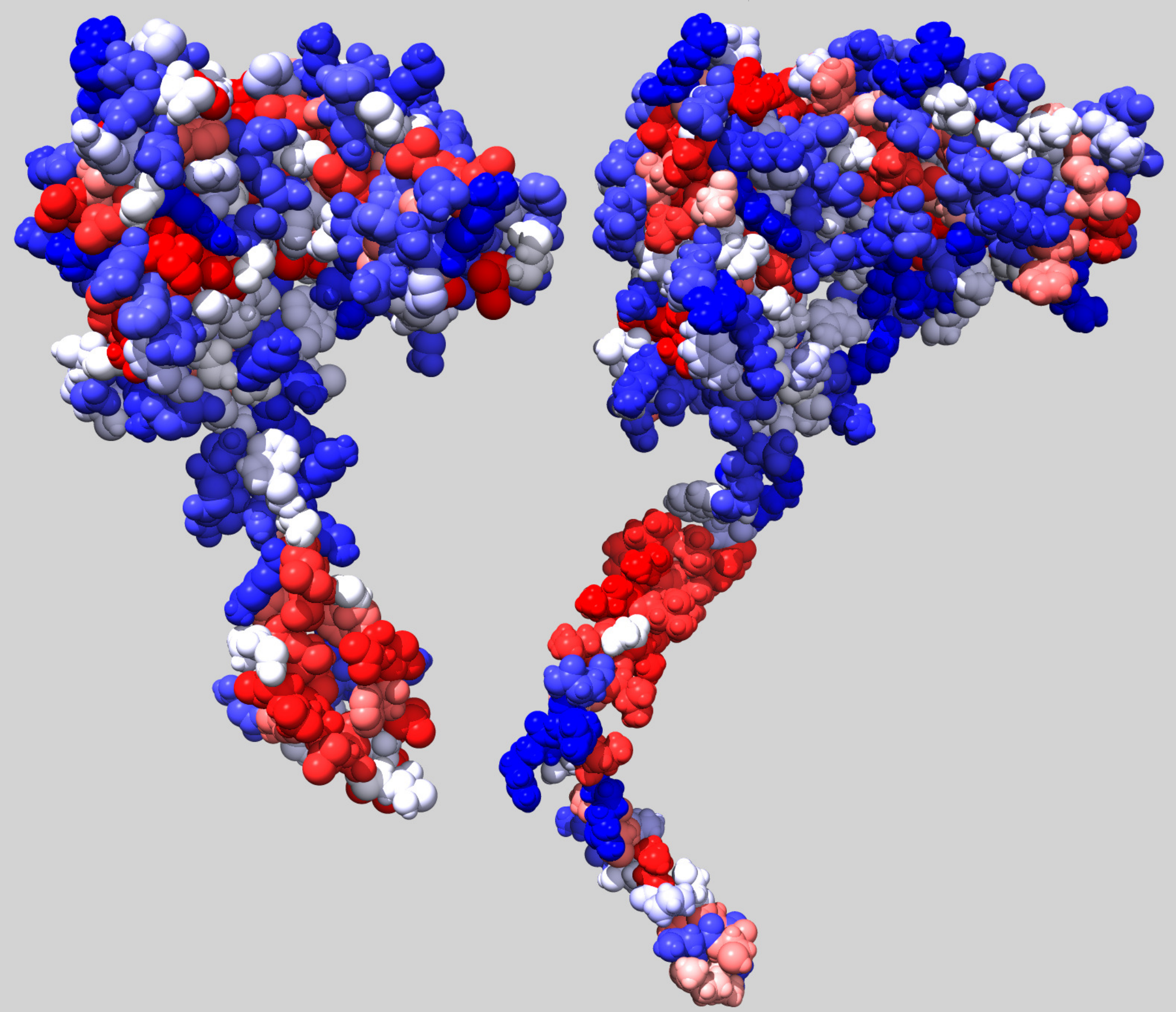


\section{Figure 3}

Structure of the NAD glycohydrolase gene from the genome of Protobothrops mucrosquamatus (Aird et al., 2017a), showing locations of the 8 exons, and the amino acid sequence of the enzyme expressed in the venom glands.

The mature venom protein comprises 303 amino acids linked by six disulfide bonds. Exon 1 actually encodes part of the 5 '-untranslated region, as well as the $\mathrm{N}$-terminus of the protein, and Exon 8 extends well beyond the stop codon, but only the mature protein sequence is shown here. Because of split arginine codons that bridge the Exon 6-7 and 7-8 boundaries, splicing out the sixth exon in the short P. mucrosquamatus NADase variants would retain the arginine after the splice. Distances between exons shown above are for purposes of illustration only, and are not proportionally scaled.
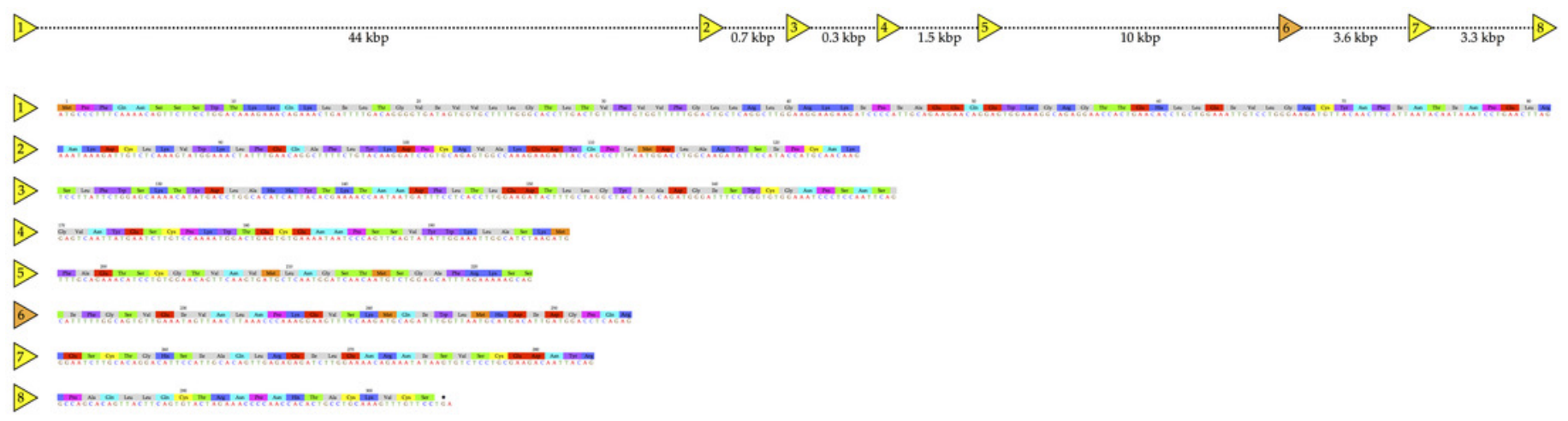


\section{Figure 4 (on next page)}

In Protobothrops mucrosquamatus, the 8-exon gene is transcribed into a long NADase and a shorter form that lacks exon 6 .

A. Its deletion results in the loss of 31 amino acids from Ser223-Gln253. With split arginine codons at both the exon 6-7 and exon 7-8 boundaries, no amino acid substitution occurs in the shortened (272-residue) structure. B. The effect of removing these 31 residues is to delete the loose helical region (Ser223-Gln253) from the center of the molecule, including the catalytic residue, Glu229, (Glu232 in Figure 1) leaving the remainder of the structure essentially intact. If this alternately spliced protein is expressed, it is difficult to imagine what its function might be. Models were created using GalaxyTBM (Ko et al. 2012). Disulfide bond formation, energy minimization, and structural manipulations were performed using Chimera 1.13 (Pettersen et al. 2004) . Amino acid classes are colored as in Figure 1. 
P. mucrosquamatus Long L L E I V L G R C Y N F I N T I N P E L R N K D C L K V W K L F E Q A F L Y K D P C R V A K E D Y Q P L M D L A R Y S I P. mucrosquamatus Short L L E I V L G R C Y N F I N T I N P E L R N K D C L K V W K L F E Q A F F L Y K D P C R V A K E D Y Q P L M D L A R Y S I P. mucrosquamatus Short P C N K S L F W S K T Y D L A H H Y T K T N N D F L T LE D T L L G Y I A D G I S W C G N P S N S G V N Y E S C P K W T 


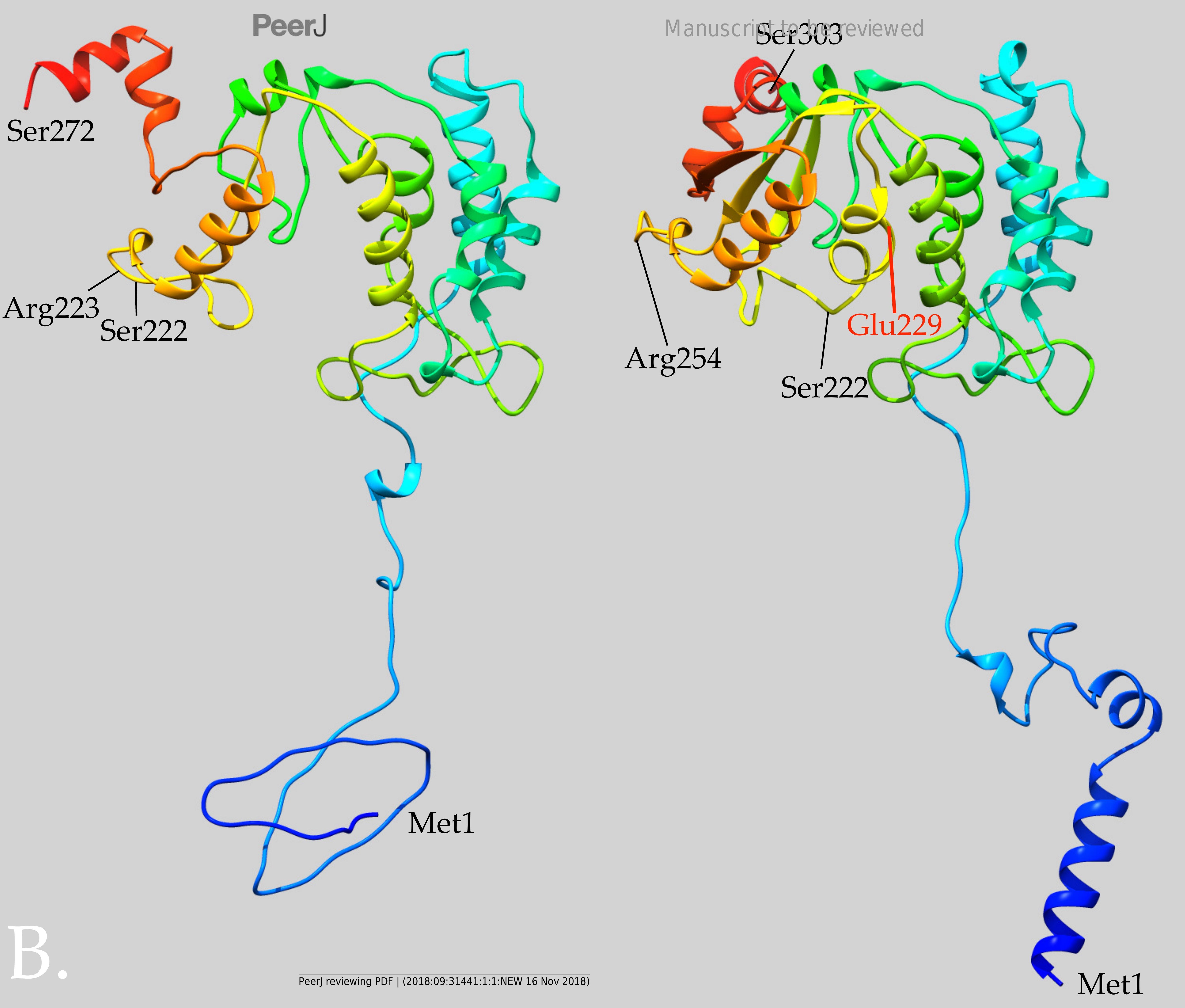




\section{Table 1 (on next page)}

Species negative for NADase activity, according to Tatsuki et al. (1975) . 
Table 1. Species negative for NADase activity, according to Tatsuki et al. (1975).

2

\begin{tabular}{|c|c|c|}
\hline Elapidae & Viperidae & Crotalidae \\
\hline Dendroaspis angusticeps & Bitis arietans & Bothrops atrox \\
\hline Dendroaspis polylepis & Bitis gabonica & Crotalus adamanteus \\
\hline Hemachatus haemachatus & Daboia russellii & Crotalus basiliscus \\
\hline Naja atra & Daboia palestinae & Crotalus durissus terrificus \\
\hline Naja haje & Echis carinatus & Crotalus viridis viridis \\
\hline Naja melanoleuca & Vipera ammodytes & Ovophis okinavensis \\
\hline Naja nivea & & Protobothrops flavoviridis \\
\hline Ophiophagus hannah & & Protobothrops mucrosquamatus \\
\hline
\end{tabular}




\section{Table 2 (on next page)}

Transcript IDs and protein accession numbers used in this study. 
Table 2. Transcript IDs and protein accession numbers used in this study.

\begin{tabular}{|c|c|c|c|c|}
\hline Taxon & Name & Scaffold ID & Gene ID & Transcript ID \\
\hline Homo sapiens & CD38 & NC_000004.12 & 952 & NM_001775.3 \\
\hline Thamnophis sirtalis & CD38 & NW_013658259 & 106543891 & XM_014059996.1 \\
\hline Python bivittatus & CD38 & NW_006532481 & 103059310 & XM_007424863.2 \\
\hline Anolis carolinensis & $\mathrm{CD} 38$ & NC_014779 & 100566030 & XM_016993323.1 \\
\hline Xenopus laevis & CD38 & NC_030724.1 & 100036901 & NM_001097679.1 \\
\hline $\begin{array}{l}\text { Protobothrops } \\
\text { mucrosquamatus }\end{array}$ & $\begin{array}{l}\text { Long } \\
\text { CD38 }\end{array}$ & NW_015387543 & 107292463 & XM_015821551.1 \\
\hline \multirow[t]{2}{*}{$\begin{array}{l}\text { Protobothrops } \\
\text { mucrosquamatus }\end{array}$} & $\begin{array}{l}\text { Short } \\
\text { CD38 }\end{array}$ & NW_015387543 & 107292463 & XM_015821552.1 \\
\hline & & Accession \# & & \\
\hline \multirow[t]{2}{*}{ Gallus gallus } & CD38 & $\begin{array}{c}\text { NCBI } \\
\text { ADQ89191.1 }\end{array}$ & & \\
\hline & & Transcript ID & & \\
\hline Micrurus carvalhoi & CD38 & $\begin{array}{c}\text { DN61384_c0_g1_i } \\
1 \text { I m.5640 }\end{array}$ & & \\
\hline Micrurus corallinus & CD38 & $\begin{array}{c}\text { DN100482_c0_g2 } \\
\text { _i2 } 1 \text { m.92 }\end{array}$ & & \\
\hline Micrurus lemniscatus & CD38 & $\begin{array}{c}\text { DN22889_c0_g1_i } \\
1 \mid \mathrm{m} .65259\end{array}$ & & \\
\hline Micrurus paraensis & CD38 & $\begin{array}{c}\text { DN86064_c0_g1_i } \\
1 \text { I m.15110 }\end{array}$ & & \\
\hline Micrurus spixii & CD38 & $\begin{array}{c}\text { DN121140_c2_g1 } \\
\text { _i1 I m.22327 }\end{array}$ & & \\
\hline Micrurus surinamensis & CD38 & $\begin{array}{c}\text { DN77054_c0_g1_i } \\
1 \mid \mathrm{m} .2918\end{array}$ & & \\
\hline
\end{tabular}




\begin{tabular}{|l|c|c|l|l|}
\hline Ovophis okinavensis & CD38 & $\begin{array}{c}\text { Oo_comp19518_c } \\
\text { 0_seq1 }\end{array}$ & & \\
\hline Protobothrops elegans & CD38 & $\begin{array}{c}\text { Pe_comp350_c0_s } \\
\text { eq1 }\end{array}$ & & \\
\hline $\begin{array}{l}\text { Protobothrops } \\
\text { flavoviridis }\end{array}$ & CD38 & $\begin{array}{c}\text { Pf_comp3789_c0_ } \\
\text { seq1 }\end{array}$ & & \\
\hline
\end{tabular}

